\title{
Muscle spindle activity in alternating tremor of Parkinsonism and in clonus ${ }^{1}$
}

\author{
K.-E. HAGBARTH ${ }^{2}$, G. WALlin, L. LÖFSTEDT, AND S.-M. AQUILONIUS
}

From the Department of Clinical Neurophysiology and Department of Neurology, University Hospital, Uppsala, Sweden

SYNOPSIS Single unit activity in spindle afferent nerve fibres from the finger flexors, the anterior tibial muscle, and the calf muscles was recorded intraneurally with tungsten microelectrodes in patients with Parkinsonism with resting tremor and in spastic patients with clonus. During tremor of Parkinsonism, involving the receptor bearing muscles, the Ia afferent fibre discharge patterns were similar to those seen previously in healthy subjects during voluntary fast alternating finger or foot movements: besides the stretch discharges occurring during the relaxation phases, discharges also occurred during the contraction phases. Such contraction discharges, presumed to originate from intrafusal muscle fibre contractions, were not seen in the spastic patients during clonus. During the clonic oscillations each afferent stretch discharge was regularly followed by a stretch reflex contraction which on its falling phase elicited a new volley of impulses in the Ia afferent fibres. The findings are considered to support the notion that, like the contractions in nor- $\theta$ mal voluntary alternating movements, the contractions in tremor of Parkinsonism are organized according to the principle of alpha-gamma coactivation, whereas the contractions in clonus are stretch reflexes causing pure alpha contractions.

Most authors agree that the resting tremor in Parkinsonism is characterized by alternating excitation of flexors and extensors, each muscle being rhythmically activated at a rate varying between 3-7 Hz (Calne, 1970; Lance, 1970). This reciprocal EMG pattern is similar to that seen in healthy subjects making fast voluntary finger or foot movements and it is also similar to that of clonus, where the frequency of the beats is generally about $5-8 \mathrm{~Hz}$.

Clonus is usually attributed to stretch reflex oscillations, and recent recordings from muscle spindle afferent nerve fibres in man support this notion by showing that the contractions in clonus cause spindle unloadings alternating with afferent stretch discharges during the relaxation phases (Szumski et al., 1974). In fast voluntary alternating movements, on the other hand, spindle discharges are seen not only during the relaxation but also during the contraction phases,

1 The investigation was supported by the Swedish Medical Research Council (Project No. B75-04X-2881-06A).

2 Reprint requests: Dr K.-E. Hagbarth, at above address.

(Accepted 18 February 1975.) indicating that, in contrast with clonus, such normal movements are organized according to the principle of alpha-gamma coactivation (Hagbarth et al., 1975). The aim of the present investigation was to study the pattern of spindle activation in the alternating tremor in Parkinsonism as well as in clonus and to compare the findings with those previously obtained during normal voluntary alternating movements.

\section{METHODS}

Seven patients with Parkinsonism with resting, alternating tremor were examined with microelectrode recordings made either from median muscle nerve fascicles at the elbow supplying the long finger flexor muscles (four patients) or from peroneal muscle nerve fascicles at the fibular head supplying the anterior tibial or long peroneal muscles (three patients). The main criterion in the selection of patients and recording sites was the strength of the tremor in the muscles concerned: fairly stable tremor of weak or moderate strength restricted to the forearm or lower leg muscles was preferred since 
the microelectrodes were easily dislodged by large oscillating movements. The age of the patients ranged from 38 to 76 years (mean 60). Five of them were under treatment with L-dopa with daily doses varying from 1.2 to $5.2 \mathrm{~g}$; the other two received no antiParkinsonism therapy.

The five spastic patients selected for the study were chosen because clonus could be easily elicited either in the foot (four patients) or in the fingers (one patient). The microelectrode recordings were made either from gastrocnemius nerve fascicles in the popliteal fossa or from finger flexor nerve fascicles at the elbow. The age of these patients ranged from 22 to 43 years (mean 34). The spasticity was due to cerebral vascular accident in two cases, to a spinal cord injury in one case and to multiple sclerosis in two cases. In none of them was there any marked sustained dystonia or contracture in the limb investigated.

The ways of assessing that the electrode had impaled a muscle nerve fascicle, and the criteria used

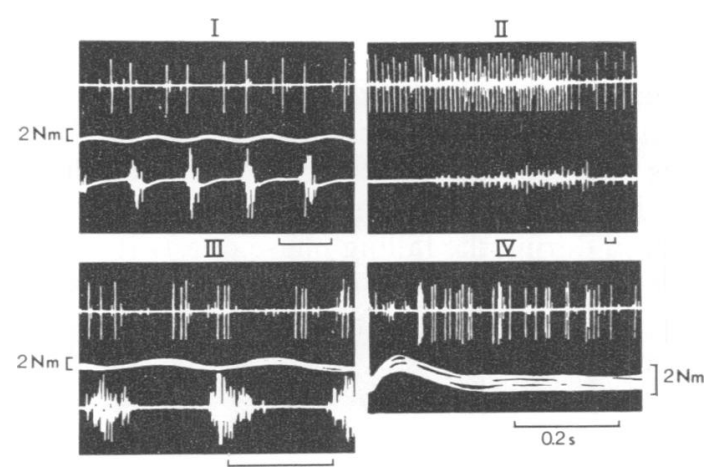

FIG. 1 I and III: contraction and relaxation discharges in a finger flexor Ia afferent unit during finger tremor in Parkinsonism, isometrically recorded. Upper traces: nerve record. Middle traces: torque at metacarpophalangeal joint (fingers kept in $20^{\circ}$ flexion). Bottom trace: EMG from finger flexors. In III three consecutive oscilloscope sweeps (phase locked to torque signal) are superimposed. II: Same unit responding to a slow passive finger extension movement of $10^{\circ}$. The movement, which is not recorded, starts at beginning of sweep (during tremor-free period) and lasts $4 \mathrm{~s}$. Unit firing (upper trace) and flexor EMG activity (bottom trace) increase during stretch movement. IV: Same unit exhibiting typical muscle spindle behaviour during electrically induced finger flexor twitches. Nerve record (upper trace) and torque (lower trace) in five superimposed oscilloscope sweeps.
TABLE

IA FIBRES SAMPLED IN PATIENTS WITH PARKINSONISM AND SPASTICITY

\begin{tabular}{lccccc}
\hline & Parkinsonism & \multicolumn{3}{c}{ Spasticity } \\
\hline Superficial and deep finger flexors & 9 & 5 & 3 & 3 \\
Anterior tibial & 9 & 4 & 0 & \\
Gastrocnemius & 0 & & 13 & 5 \\
\hline Total & 18 & 9 & 16 & 8 \\
\hline
\end{tabular}

Italic figures indicate the numbers of units studied during ongoing tremor or clonus.

for identifying single Ia afferent fibres have previously been described (Hagbarth et al., 1975). Most of the recordings were of the multi-unit type, but the majority of experiments also included some successful single unit recordings from muscle spindle afferent fibres. The Table shows the total number of
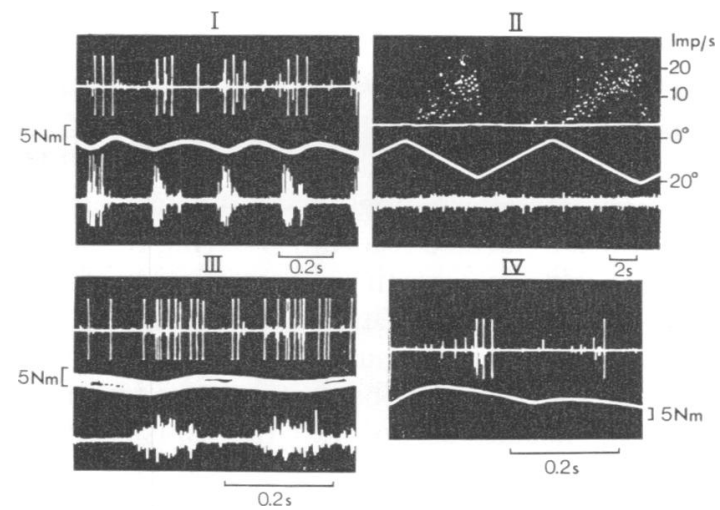

FIG. 2 I and III: Contraction- and relaxation discharges in a Ia afferent unit from the anterior tibial muscle during foot tremor in Parkinsonism, isometrically recorded. Upper traces: nerve records. Middle traces: ankle joint torque (foot at right angles to tibia). Bottom trace: EMG from anterior tibial muscle. In III four consecutive oscilloscope sweeps (phase locked to torque signal) are superimposed. II: same unit responding to slow passive movements of ankle joint during tremor-free period. Upper trace: instantaneous frequency plot of unit discharge. Middle trace: ankle joint angle. $0^{\circ}$ denotes foot at right angle to tibia. Downward deflections = plantar movements . Bottom trace: EMG from anterior tibial muscle. IV: Same unit exhibiting typical muscle spindle behaviour during electrically induced twitch of anterior tibial muscle. Nerve recorded in upper and torque in bottom trace. 
primary spindle afferent fibres sampled from various muscles in patients with Parkinsonism and spasticity.

The general experimental situation, the electrodes, transducers, recording and display systems were the same as described in the previous paper. Thus, besides the neural activity the following signals and events were recorded: (1) the EMG activity from the receptor-bearing muscle, recorded with needle or surface electrodes (sometimes with additional recordings from neighbouring muscles); (2) strain gauge signals indicating the torque developed either by the finger flexors and extensors in their actions on the metacarpophalangeal joints, or by the calf and peroneal muscles in their action as dorsi- and plantar flexors of the foot; (3) goniometer signals indicating the angular position of the metacarpophalangeal joints or of the ankle, the other joints of the extremity being fixed in extended position. As a rule the tremor and clonus were recorded isometrically with the ankle and metacarpophalangeal joints fixed in a 'mid-position' and with the subjects trying to remain passive and relaxed.

\section{RESULTS}

PARKINSONISM TREMOR In both finger and foot tremor simultaneous EMG recordings from flexors and extensors showed reciprocal EMG patterns with alternating bursts of activity in the antagonists. Surface recordings during tremor of large amplitude sometimes gave the impression of a doubling of the tremor frequency (from about 5 to $10 \mathrm{~Hz}$ ) with a change from reciprocal to synchronous activation, but as judged by more selective needle recordings this was a false impression caused by artificial 'overhearing' from distant muscle groups (Calne and Lader, 1969). In some patients with finger tremor it was also observed that the finger flexors could be activated either in- or out-of-phase with wrist flexors and/or pronators, and when the out-ofphase patterns occurred surface recordings could easily give misleading impressions of the timing of the finger flexor contractions.

Three main types of Ia fibre discharge patterns were observed during tremor involving the receptor bearing muscles: (1) activation occurring twice during each cycle-that is, once during the contraction and once during the relaxation phase; (2) activation occurring only during the contraction phases; (3) activation occurring only during the relaxation phases. For any particular unit spontaneous transition from one type of discharge pattern to another was frequently seen, presumably depending on small random variations in the spatial distribution of the extrafusal contractions relative to the site of the receptor. The majority of the units (seven out of nine) exhibited patterns of the first and second type only, whereas the third type of pattern was exhibited during short periods by two units (one anterior tibial and one finger flexor unit). Since only surface EMG recordings were made in these two cases, it cannot be excluded that, during the periods concerned, the tremor in the receptor bearing muscles declined or ceased while neighbouring muscles or muscle portions temporarily took over as prime generators of the mechanical oscillations.

Figures 1 and 2 show examples of spindle discharge patterns of types 1 and 2. The finger flexor unit in Fig. 1 fired once during each contraction and once during each relaxation phase, whereas the anterior tibial unit in Fig. 2 responded with a burst of impulses during each contraction phase, with only occasional single discharges occurring during the relaxation. During electrically induced muscle twitches both units showed the typical spindle behaviourthat is, they paused during the rising and dis charged during the falling phase of a twitch. For units responding with impulse volleys during both the tremor contraction and relaxation phases, the two types of volleys tended to

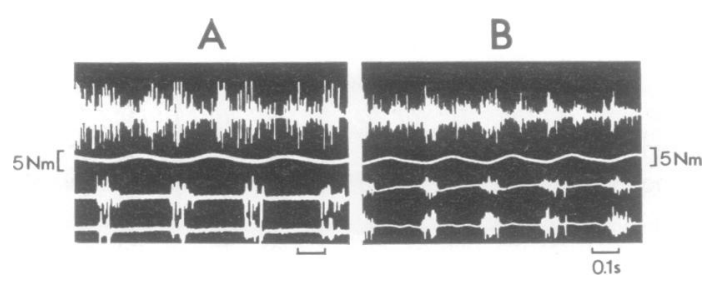

FIG. 3 Contraction and relaxation discharges seen in multi-unit recordings from muscle nerve fascicles during Parkinsonism tremor. A. Finger tremor. Upper trace: activity in median nerve fascicle to finger flexors. Second trace: torque in metacarpophalangeal joints. Bottom two traces: EMG from deep and superficial finger flexors. B. Foot tremor. Upper trace: activity in peroneal nerve fascicle innervating anterior tibial muscle. Second trace: torque in ankle joint. Bottom two traces: EMG led off distally and proximally from the anterior tibial muscle. 
merge, especially when the tremor frequency was high, and it was then often difficult to tell whether spikes in the 'merging zone' should be classified as contraction impulses or not. When no relaxation discharges were present, however, it could be seen that the initial spindle contraction impulses regularly appeared with some delay $(5-30 \mathrm{~ms})$ after the start of the EMG bursts.

Since showers of impulses in spindle afferent fibres usually stand out quite clearly in multiunit recordings from muscle nerves (Wallin et al., 1973; Hagbarth et al., 1975) such recordings can also give clues about the spindle discharge patterns during tremor. In all such composite muscle nerve neurograms, obtained during tremor, a grouping of nerve impulses was seen that agreed well with the spindle discharge patterns as seen in the single unit recordings. As illustrated in Fig. 3 two rather distinct showers of neural impulses appeared during each tremor

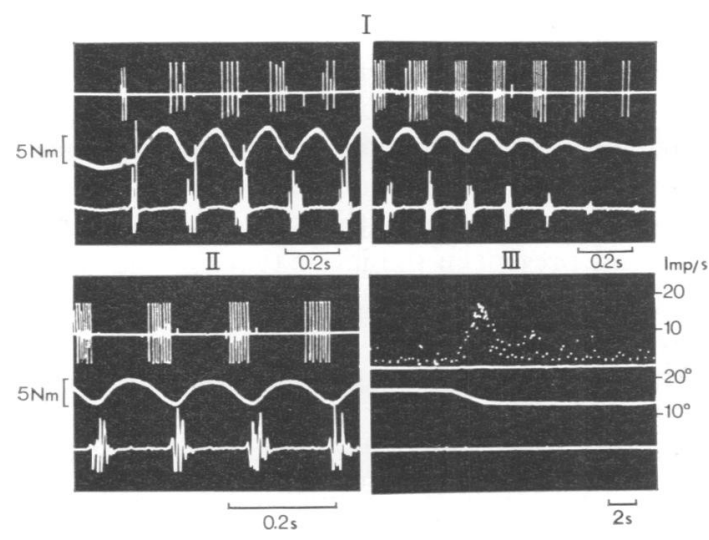

FIG. 4 I and II: relaxation discharges in a Ia afferent unit from medial gastrocnemius muscle during ankle clonus in hemiplegic patient. Upper traces: nerve record. Second traces: torque in ankle joint. Bottom traces: EMG from medial gastrocnemius muscle. In I the clonus is started by an Achilles tendon tap seen as initial small deflexion in torque record (left), about $10 \mathrm{~s}$ later the clonus gradually subsides (right). II shows middle part of clonus sequence on expanded time scale. III: same unit responding to slow passive stretch of calf muscles. Upper trace: instantaneous frequency plot of unit discharge. Second trace: angle at ankle joint. Calf muscle stretch drawn downwards. Bottom trace: EMG from calf muscles. cycle, one during the contraction phase and one during the phase of relaxation. No lidocaine nerve infiltrations were made to check whether gamma efferent nerve fibre blockade could eliminate the neural contraction discharges in a manner similar to that previously demonstrated in healthy subjects during voluntary alternating movements.

CLONUS All units identified as primary spindle afferent fibres showed the same type of firing pattern when clonus was initiated in the receptor bearing muscle. They all responded to the clonic beats in a similar way as to electrically induced muscle twitches-that is, they were silent during the contraction phases and fired during the phases of relaxation. Figure 4 shows an example of this spindle behaviour during calf muscle clonus, initiated by a sudden moderate tap on the Achilles tendon. As judged by the spindle firing pattern and the time relationship between the afferent and motor events, the clonus may be attributed to oscillations in the stretch reflex servo loop. In most of the single units recordings, however, no positive correlation was observed between the number or frequency of the spindle impulses per relaxation phase and the strength of the succeeding reflex EMG response.

\section{DISCUSSION}

There is now a great deal of evidence indicating that the tremor of Parkinsonism in man and Parkinsonism-like tremor in monkeys and cats is not critically dependent on peripheral sensory feed-back, even though such feed-back can modify the amplitude and phasing of the tremor bursts (for references, see Alberts, 1972). There is also strong evidence that the ventrolateral nucleus of the thalamus and the corticospinal pathways are involved in the generation of descending volleys which ultimately drive the alpha motor neurones to produce tremor (for references, see Calne, 1970). Alberts (1972) suggests that 'a program of motor behaviour for the voluntary production of rapid alternating movements may be stored in the somatomotor cortex, and that Parkinsonian tremor may be the result of involuntary activation of this program'. 
ALPHA-GAMMA LINKAGE PRESERVED IN TREMOR AND RIGIDITY The present findings indicate that, in contrast with electrically induced muscle twitches and clonic contractions, which cause unloading of spindles, tremor contractions are accompanied by spindle acceleration. In this respect, they are similar to the contractions in rapid voluntary alternating movements (Hagbarth et al., 1975). In both cases, spindle acceleration can be taken as evidence for a rhythmical fusimotor outflow occurring in parallel with and directed towards the same muscle groups as the outflow in the alpha motor nerve fibres. As judged by previous muscle spindle recordings in patients with Parkinsonism, the rigidity involves a fusimotor coactivation similar to that observed during voluntary sustained contractions in healthy subjects (Wallin et al., 1973). This, in combination with the present findings, supports the notion that the alpha-gamma linkage is retained both in rigidity and tremor.

REFLEX FUNCTIONS OF SPINDLE DISCHARGES DURING TREMOR In tremor, as in voluntary alternating movements, the EMG bursts started before the initiation of the spindle contraction discharges, and thus it can be concluded that the indirect loop through the spindles plays no part in the initiation of individual tremor beats. On the other hand, the spindle contraction discharges started early enough to permit an autogenetic excitatory influence on the later parts of individual tremor bursts and also to impose a properly timed reciprocal inhibition upon antagonistic motor neurones. Thus, like normal voluntary contractions, tremor contractions are not 'servo driven' but may be 'servo assisted' by the stretch reflex (Matthews, 1972). The present results, however, give no clues concerning the power of this servo-assistance. It may, for instance, be compensated for by opposing reflex effects induced by spindle relaxation discharges from antagonistic muscles.

In tremor, there was no fixed interval between spindle relaxation discharges and succeeding EMG bursts. When the tremor rhythm was slow $(3-5 \mathrm{~Hz})$ this interval often exceeded $150 \mathrm{~ms}$ (Fig. 2), but with higher tremor frequencies the interval sometimes fell below $100 \mathrm{~ms}$. At any rate, the intervals observed exceeded the latency of the spinal stretch reflex in the muscles concerned, and thus the recurrent contractions in tremor differ from those in clonus (see below) in that they cannot be explained in terms of stretch reflex oscillations. This, however, does not exclude the possibility that the spindle relaxation discharges in tremor may exert some rhythmical drive on the alpha motoneurones, facilitating such centrally driven movements in which the rhythm is high enough to correspond to the 'period of revolution' for the stretch reflex.

SPINDLE FIRING IN CLONUS As regards the pattern of spindle discharge during clonus, the present results agree with those of Szumski et al. (1974) and with the notion that clonus is maintained by reverberating stretch reflexes elicited by preceding spindle relaxation discharges. The present data, however, do not lend support to the claims of Szumski et al. (1974) that in patients with clonus the spindles exhibit signs of increased dynamic sensitivity (cf. Jacobi et al., 1970). It is true that in some clonus sequences the spindle afferent fibres temporarily reached firing rates of about $150 \mathrm{~Hz}$ (see Fig. 4), and relaxation discharges of such strength have so far not been observed during tremor or during rapid volun tary alternating movements. The significance of this finding is obscure, however. It may be argued, for instance, that the contraction discharges present in the latter types of movements made the spindles less sensitive to the intervening stretch stimuli during the relaxation phases. Also, quantitative comparisons of this type were hampered by the fact that, for any particular unit, the number and frequency of impulses per relaxation phase varied greatly from one tremor or clonus sequence to the next, presumably depending on small random changes in the spatial distribution of the contractions relative to the site of the receptor. In a study where standard ramp stretches were used as test stimuli for spindles in the calf muscles, no significant difference in dynamic spindle sensitivity was observed in spastic as compared with healthy subjects (Hagbarth et al., 1973), and the unit illustrated in Fig. 4 did not fire more vigorously to passive stretch than did many normal spindle primary endings that were previously studied. Until further studies with standard ramp stretches have been made in different forms of 
spasticity, it remains uncertain whether an increased dynamic fusimotor drive plays an important role in the development of clonus. Internuncial neurone excitability changes in the spinal cord, loss of presynaptic inhibition on the Ia fibre terminals, and central sprouting are other plausible causes of the exaggerated dynamic stretch reflexes which, in turn, are responsible for the clonic oscillations.

\section{REFERENCES}

Alberts, W. W. (1972). A simple view of Parkinsonian tremor. Electrical stimulation of cortex adjacent to the Rolandic fissure in awake man. Brain Research, 44, 357-369.

Calne, D. B., and Lader, M. H. (1969). Electromyographic studies of tremor using an averaging computer. Electroencephalography and Clinical Neurophysiology, 26, 86-92.

Calne, D. B. (1970). Parkinsonism: Physiology, Pharmacology, and Treatment. Arnolds: London.

Hagbarth, K.-E., Wallin, G., and Löfstedt, L. (1973).
Muscle spindle responses to stretch in normal and spastic subjects. Scandinavian Journal of Rehabilitation Medicine, 5, 156-159.

Hagbarth, K.-E., Wallin, B. G., and Löfstedt, L. (1975). Muscle spindle activity in man during voluntary fast alternating movements. Journal of Neurology, Neurosurgery, and Psychiatry, 38, 625-635.

Jacobi, H. M., Krott, H. M., and Poremba, M. B. (1970). Recording of proprioceptive muscle afferents in man by various disorders of tendon reflex. Electromyography (Louvain), 10, 307-316.

Lance, J. W. (1970). A Physiological Approach to Clinical Neurology. Butterworths: London.

Matthews, P. B. C. (1972). Mammalian Muscle Receptors and their Central Actions, pp. 546-611. Arnold: London.

Szumski, A. J., Burg, D , Struppler, A., and Velho, F. (1974). Activity of muscle spindles during muscle twitch and clonus in normal and spastic human subjects. Electroencephalography and Clinical Neurophysiology, 37, 589-597.

Wallin, B. G., Hongell, A., and Hagbarth, K.-E. (1973). Recordings from muscle afferents in Parkinsonian rigidity. In New Developments in Electromyography and Clinical Neurophysiology, vol. 3, pp. 263-272. Edited by J. E. Desmedt. Karger: Basel. 